\title{
Coherent states for quadratic Hamiltonians
}

\author{
Alonso Contreras-Astorga, David J. Fernández C. and Mercedes Velázquez \\ Departamento de Física, Cinvestav, A.P. 14-740, 07000 México D.F., Mexico
}

\begin{abstract}
The coherent states for a set of quadratic Hamiltonians in the trap regime are constructed. A matrix technique which allows to identify directly the creation and annihilation operators will be presented. Then, the coherent states as simultaneous eigenstates of the annihilation operators will be derived, and they are going to be compared with those attained through the displacement operator method. The corresponding wave function will be found, and a general procedure for obtaining several mean values involving the canonical operators in these states will be described. The results will be illustrated through the asymmetric Penning trap.
\end{abstract}

PACS: 03.65.Ge, 03.65.Sq, 37.10.Ty, 37.30.+i

\section{Introduction}

As it was shown by Schrödinger in 1926 for the harmonic oscillator, the quasi-classical states are important for the description of physical systems in the classical limit (see e.g. [1]). The catchy term coherent states (CS) was used for the first time by Glauber long after, when studying electromagnetic correlation functions [2,3]. With this application it was realized that the CS are useful as well in the intrinsically quantum domain. Indeed, the CS approach is nowadays widely employed for dealing with quantum physical systems. According to Glauber there are three equivalent ways to construct the CS for the harmonic oscillator. The first one is to define them as eigenstates of the annihilation operator. The second one is to build the CS through the action of a displacement operator onto the ground state. The third way is to consider them as quantum states having a minimum Heisenberg uncertainty relationship. These three properties can be used as definitions to build the CS for systems different from the harmonic oscillator. However, it is noteworthy that each of them leads to sets of CS which do not coincide in general [4-13]. In fact, even for the harmonic oscillator the third definition does not produce just the standard CS, since it includes as well the so-called squeezed states [14,15].

In spite of its long term life, from time to time there are some advances which maintain the subject alive. This is the case, e.g., of the recently discovered coherent states for a charged particle inside an ideal Penning trap [16]. Since the corresponding Hamiltonian is quadratic in the position and momentum operators, one would expect that the CS appear as a generalized displacement operator acting onto the corresponding ground state. However, it is worth to notice that the Penning trap Hamiltonian does not have any ground state at all, since it is not a positively 
defined operator. Despite, it was possible to implement in a simple way the corresponding CS construction. Thus, we need to take into account this Hamiltonian property when studying the coherent states of general systems.

In this article we are going to address the CS construction for systems characterized by a certain set of quadratic Hamiltonians. The CS will be built up as simultaneous eigenstates of the corresponding annihilation operators, and also by applying a generalized displacement operator onto an appropriate extremal state. We will see that in the case of a positively defined Hamiltonian this extremal state will coincide with the ground state. In order to perform the CS construction, we need to find first the annihilation and creation operators. This task will be done by using a matrix technique, which generalizes the one employed in [16] (see also [17- -20]). In this way, we will simply and systematically identify the characteristic algebra of the involved Hamiltonians. Our procedure represents a generalization to dimensions greater than one of the standard technique to deal with the harmonic oscillator, which is closely related to the well known factorization method (see, e.g., [21,22]).

The paper is organized as follows. In section 2 we will introduce a detailed recipe for systematically obtaining the annihilation and creation operators for quadratic Hamiltonians in the trap regime. The coherent states derivation shall be elaborated in section 3 , while in section 4 we will address the completeness of this set of CS, we shall obtain the mean values of several important physical quantities and the time evolution of these states. We are going to apply our general results to an asymmetric Penning trap in section 5, and our conclusions will be presented at section 6 .

\section{Ladders operators for quadratic Hamiltonians}

Along this work we are going to consider a general set of $n$-dimensional quadratic Hamiltonians of the form

$$
H=\frac{1}{2} \eta^{T} \mathbf{B} \eta
$$

where $\mathbf{B}$ is a $2 n \times 2 n$ real constant symmetric matrix, $\eta=(\vec{X}, \vec{P})^{T}$, and $\vec{X}, \vec{P}$ are the $n$-dimensional coordinate and momentum operators in the Schrödinger picture satisfying the canonical commutation relationships $\left[X_{i}, P_{j}\right]=i \delta_{i j}$ (notice that a system of units such that $\hbar=1$ will be used throughout this paper). The time evolution of the operator vector $\eta(t)=$ $U^{\dagger}(t) \eta U(t)$ in the Heisenberg picture is governed by

$$
\frac{d \eta(t)}{d t}=U^{\dagger}(t)[i H, \eta] U(t)=U^{\dagger}(t) \boldsymbol{\Lambda} \eta U(t)=\boldsymbol{\Lambda} \eta(t),
$$

$U(t)$ being the evolution operator of the system such that $U(0)=\mathbf{1}$, and $\boldsymbol{\Lambda}=\mathbf{J B}$, where $\mathbf{J}$ is the well known $2 n \times 2 n$ matrix

$$
\mathbf{J}=\left(\begin{array}{cc}
0 & \mathbf{1}_{n} \\
-\mathbf{1}_{n} & 0
\end{array}\right)
$$

satisfying

$$
\mathbf{J}^{T}=-\mathbf{J}, \quad \mathbf{J}^{2}=-\mathbf{1}_{2 n}, \quad \operatorname{det}(\mathbf{J})=1,
$$

in which $\mathbf{1}_{m}$ represents the $m \times m$ identity matrix. The solution of Eq.(2) is given by

$$
\eta(t)=e^{\boldsymbol{\Lambda} t} \eta(0)=e^{\boldsymbol{\Lambda} t} \eta .
$$


In order to identify the annihilation and creation operators of $H$, we need to find the right and left eigenvectors of $\Lambda$. Since in general $\Lambda$ is non-hermitian, its right and left eigenvectors are not necessarily adjoint to each other.

Let us consider in the first place the $2 n$-th order characteristic polynomial of $\Lambda, P(\lambda)=$ $\operatorname{det}(\boldsymbol{\Lambda}-\lambda)=\operatorname{det}(\mathbf{J B}-\lambda)$. Using Eqs.(4) and the fact that $\mathbf{B}$ is a symmetric matrix, we obtain

$$
P(\lambda)=\operatorname{det}(\mathbf{J B}-\lambda)=\operatorname{det}\left[(\mathbf{J B}-\lambda)^{T}\right]=\operatorname{det}(\mathbf{J B}+\lambda)=P(-\lambda) .
$$

This means that if $\lambda$ is an eigenvalue of $\Lambda$, then $-\lambda$ also will be. Throughout this work we are going to denote the eigenvalues of $\Lambda$ as $\lambda_{k}$ and $-\lambda_{k}$, taking $\lambda_{k}$ in the way

$$
\operatorname{Re}\left(\lambda_{k}\right)>0 \text { or } \operatorname{Im}\left(\lambda_{k}\right)>0 \text { if } \operatorname{Re}\left(\lambda_{k}\right)=0, \quad k=1, \ldots, n
$$

Let us label as $u_{k}^{ \pm}$and $f_{k}^{ \pm}$the right and left eigenvectors associated to the eigenvalues $\pm \lambda_{k}$ respectively, i.e.,

$$
\boldsymbol{\Lambda} u_{k}^{ \pm}= \pm \lambda_{k} u_{k}^{ \pm}, \quad f_{k}^{ \pm} \boldsymbol{\Lambda}= \pm \lambda_{k} f_{k}^{ \pm} .
$$

Notice that both $u_{k}^{ \pm}$and $f_{k}^{ \pm}$can be determined from Eq.(8) up to arbitrary factors. Part of this arbitrariness will be eliminated by imposing two requirements. The first one is that the right and left eigenvectors be dual to each other, namely,

$$
f_{j}^{r} u_{k}^{r^{\prime}}=\delta_{j k} \delta_{r r^{\prime}}
$$

with $j, k=1, \ldots, n$ and $r, r^{\prime}=+,-$. The second condition, which is needed in order to recover the standard annihilation and creation operators for the one-dimensional harmonic oscillator, is to ask that the left eigenvectors $f_{k}^{ \pm}$involved in the commutators

$$
\left[f_{k}^{-} \eta, f_{k}^{+} \eta\right]=\gamma_{k}, \quad \gamma_{k} \in \mathbb{C}
$$

are such that

$$
\left|\gamma_{k}\right|=1 \text {. }
$$

In this paper we are going to discuss just the case in which there are no degeneracies in the eigenvalues $\pm \lambda_{k}$ so that the identity matrix $\mathbf{1}_{2 n}$ can be expanded as (see [23])

$$
\mathbf{1}_{2 n}=\sum_{k=1}^{n}\left(u_{k}^{+} \otimes f_{k}^{+}+u_{k}^{-} \otimes f_{k}^{-}\right),
$$

with $\otimes$ representing tensor product. Then we get

$$
\begin{aligned}
\eta(t) & =e^{\boldsymbol{\Lambda} t}\left[\sum_{k=1}^{n}\left(u_{k}^{+} \otimes f_{k}^{+}+u_{k}^{-} \otimes f_{k}^{-}\right)\right] \eta=\sum_{k=1}^{n}\left[e^{\lambda_{k} t} u_{k}^{+} \otimes f_{k}^{+} \eta+e^{-\lambda_{k} t} u_{k}^{-} \otimes f_{k}^{-} \eta\right] \\
& =\sum_{k=1}^{n}\left(e^{\lambda_{k} t} u_{k}^{+} L_{k}^{+}+e^{-\lambda_{k} t} u_{k}^{-} L_{k}^{-}\right)
\end{aligned}
$$

where $L_{k}^{ \pm} \equiv f_{k}^{ \pm} \eta$. 
It is worth to point out that, in the classical case, $L_{k}^{ \pm}$represent $c$-numbers which are related with the initial conditions. The time dependence of $\eta(t)$ is determined essentially by the $\lambda_{k}$ values, which are complex in general. If $\operatorname{Re}\left(\lambda_{k}\right) \neq 0$ it can be seen that one of the two involved exponentials of the $k$-th term in the previous relation diverges as $t$ increases and, thus, the classical motion will be in general unbounded (see [24-27]). The only way in which this does not happen is that all the eigenvalues be purely imaginary so that the corresponding exponentials will just induce oscillations in time, and therefore in this case the classical evolution of the vector $\eta(t)$ will remain always bounded.

On the other hand, in the quantum regime the $L_{k}^{ \pm}$are linear operators in the canonical variables $\vec{X}, \vec{P}$. It is straightforward to show that their commutators with $H$ reduce to

$$
\left[H, L_{k}^{ \pm}\right]=\mp i \lambda_{k} L_{k}^{ \pm}
$$

In addition, it turns out that

$$
\left[L_{j}^{-}, L_{k}^{-}\right]=\left[L_{j}^{+}, L_{k}^{+}\right]=0, \quad\left[L_{j}^{-}, L_{k}^{+}\right]=0, \quad k \neq j .
$$

However,

$$
\left[L_{k}^{-}, L_{k}^{+}\right]=\gamma_{k} \neq 0, \quad k=1, \ldots, n .
$$

Eq. (14) implies that $L_{k}^{ \pm}$behave, at least formally, as ladders operators for the eigenvectors of $H$, changing its eigenvalues by $\mp i \lambda_{k}$. However, this statement has to be managed carefully since it could happen that the action of $L_{k}^{ \pm}$onto an eigenvector of $H$ produces something which does not belong to the domain of $H$, and in this case we would not get new eigenvectors of $H$. In the next section we will explore an interesting situation (the trap regime of our systems) for which the application of $L_{k}^{ \pm}$onto an eigenvector of $H$ produce a new one with different eigenvalue.

Let us point out that Eqs. 14 16) imply that $H$ can be expressed in a simple way in terms of $\left\{L_{k}^{ \pm}, k=1, \ldots, n\right\}$ [24]. This is a consequence of the following general theorem:

Theorem. If $\mathcal{L}$ is an irreducible algebra of operators generated by $L_{i}^{ \pm}$which obey $\left[L_{i}^{+}, L_{j}^{+}\right]=$ $\left[L_{i}^{-}, L_{j}^{-}\right]=0,\left[L_{i}^{-}, L_{j}^{+}\right]=\gamma_{i} \delta_{i j}$ with $\left|\gamma_{i}\right|=1, i, j=1, \ldots, n$, then an operator $H \in \mathcal{L}$ which fulfills relation (14) can be written as

$$
H=\sum_{k=1}^{n}\left(\frac{-i \lambda_{k}}{\gamma_{k}}\right) L_{k}^{+} L_{k}^{-}+g_{0}
$$

where $g_{0} \in \mathbb{C}$.

Demonstration. Actually, due to Eqs.(14,16) it turns out that

$$
g_{0} \equiv H-\sum_{k=1}^{n}\left(\frac{-i \lambda_{k}}{\gamma_{k}}\right) L_{k}^{+} L_{k}^{-}
$$

commutes with $L_{k}^{ \pm}$for all $k$, thus with any function of them, and so $g_{0}$ must be a $c$-number.

From now on we are going to discard situations such that $\operatorname{Re}\left(\lambda_{k}\right) \neq 0$ for some $k=1, \ldots, n$, restricting ourselves to cases in which the $\lambda_{k}$ are purely imaginary for all $k$, i.e., we stick to the trap regime of our systems. 


\subsection{Algebraic structure of $H$ in the trap regime}

Let us suppose that $\lambda_{k}=i \omega_{k}$ with $\omega_{k}>0, k=1, \ldots, n$. Hence, $\lambda_{k}^{*}=-\lambda_{k}$, and since $\boldsymbol{\Lambda}$ is real, without loosing generality we can choose

$$
f_{k}^{-}=\left(f_{k}^{+}\right)^{*}, \quad u_{k}^{-}=\left(u_{k}^{+}\right)^{*} .
$$

Since $L_{k}^{ \pm}$are linear combinations of the hermitian components of $\eta\left(X_{1}, \ldots, X_{n}, P_{1}, \ldots, P_{n}\right)$, it turns out that

$$
\left(L_{k}^{ \pm}\right)^{\dagger}=L_{k}^{\mp} .
$$

Note moreover that $\gamma_{k}^{*}=\gamma_{k}$, i.e., $\gamma_{k} \in \mathbb{R}$, and the use of Eq.(11) implies that $\gamma_{k}= \pm 1$. Summarizing these results, Eq.(14) becomes in this case

$$
\left[H, L_{k}^{ \pm}\right]= \pm \omega_{k} L_{k}^{ \pm},
$$

i.e., the modifications suffered by the eigenvalues of $H$ through the action of the ladder operators $L_{k}^{ \pm}$are given by the real quantities $\pm \omega_{k}$. In addition, $H$ is factorized as (see Eq.(17))

$$
H=\sum_{k=1}^{n} \gamma_{k} \omega_{k} L_{k}^{+} L_{k}^{-}+g_{0}, \quad g_{0} \in \mathbb{R} .
$$

Notice that the previous summation either involves terms for which $\gamma_{k}=1$, which are of the oscillator kind since they are positively defined, or terms with $\gamma_{k}=-1$ which are of the antioscillator type since they are negatively defined. Thus, it is natural to define a global algebraic structure for our system (see, e.g., [16, 28]), which is independent of the spectral details but has to do with the fact that any quadratic Hamiltonian in the trap regime can be expressed in terms of several independent oscillators, some of them indeed being anti-oscillators (compare Eq.(21)). This global structure is characterized mathematically by identifying $n$ sets of number, annihilation and creation operators of the system, $\left\{N_{k}, B_{k}, B_{k}^{\dagger}\right\}, k=1, \ldots, n$, in the way:

$$
\begin{array}{ll}
B_{k}=L_{k}^{-}, & B_{k}^{\dagger}=L_{k}^{+}, \quad \text { for } \quad \gamma_{k}=1, \\
B_{k}=L_{k}^{+}, & B_{k}^{\dagger}=L_{k}^{-}, \quad \text { for } \quad \gamma_{k}=-1, \\
N_{k}=B_{k}^{\dagger} B_{k}, & k=1, \ldots, n,
\end{array}
$$

so that the standard commutation relationships are satisfied,

$$
\begin{aligned}
{\left[B_{j}, B_{k}^{\dagger}\right]=\delta_{j k}, \quad\left[B_{j}, B_{k}\right]=\left[B_{j}^{\dagger}, B_{k}^{\dagger}\right]=0, } \\
{\left[N_{k}, B_{k}\right]=-B_{k}, \quad\left[N_{k}, B_{k}^{\dagger}\right]=B_{k}^{\dagger}, \quad j, k=1, \ldots, n . }
\end{aligned}
$$

Let us construct now a basis $\left\{\left|n_{1}, \ldots, n_{n}\right\rangle, n_{j}=0,1,2, \ldots, j=1,2, \ldots, n\right\}$ of common eigenstates of $\left\{N_{1}, \ldots, N_{n}\right\}$ (the Fock states)

$$
N_{j}\left|n_{1}, \ldots, n_{n}\right\rangle=n_{j}\left|n_{1}, \ldots, n_{n}\right\rangle, \quad j=1, \ldots, n,
$$

starting from an extremal state $|0, \ldots, 0\rangle$, which is annihilated simultaneously by $B_{1}, \ldots, B_{n}$ :

$$
B_{j}|0, \ldots, 0\rangle=0, \quad j=1, \ldots, n .
$$


If we assume that $|0, \ldots, 0\rangle$ is normalized, it turns out that:

$$
\left|n_{1}, \ldots, n_{n}\right\rangle=\frac{B_{1}^{\dagger n_{1}} \ldots B_{n}^{\dagger n_{n}}|0, \ldots, 0\rangle}{\sqrt{n_{1} ! \cdots n_{n} !}} .
$$

Moreover, $B_{j}$ and $B_{j}^{\dagger}, j=1, \ldots, n$, act onto $\left|n_{1}, \ldots, n_{n}\right\rangle$ in a standard way:

$$
\begin{aligned}
B_{j}\left|n_{1}, \ldots, n_{j-1}, n_{j}, n_{j+1}, \ldots, n_{n}\right\rangle & =\sqrt{n_{j}}\left|n_{1}, \ldots, n_{j-1}, n_{j}-1, n_{j+1}, \ldots, n_{n}\right\rangle, \\
B_{j}^{\dagger}\left|n_{1}, \ldots, n_{j-1}, n_{j}, n_{j+1}, \ldots, n_{n}\right\rangle & =\sqrt{n_{j}+1}\left|n_{1}, \ldots, n_{j-1}, n_{j}+1, n_{j+1}, \ldots, n_{n}\right\rangle .
\end{aligned}
$$

Now, in terms of the operators $\left\{B_{j}, B_{j}^{\dagger}, j=1, \ldots, n\right\}$ our Hamiltonian is expressed by

$$
H=\sum_{k=1}^{n} \gamma_{k} \omega_{k} B_{k}^{\dagger} B_{k}+g_{0}^{\prime}
$$

It is clear that the Fock states $\left|n_{1}, \ldots, n_{n}\right\rangle$ are eigenstates of $H$ with eigenvalues $E_{n_{1}, \ldots, n_{n}}=$ $\gamma_{1} \omega_{1} n_{1}+\ldots+\gamma_{n} \omega_{n} n_{n}+g_{0}^{\prime} \equiv E\left(n_{1}, \ldots, n_{n}\right)$. In particular, the extremal state $|0, \ldots, 0\rangle$ has eigenvalue $E_{0, \ldots, 0}=g_{0}^{\prime}$. In case that $\gamma_{k}=1$ for all $k$, then $H-g_{0}^{\prime}$ will be a positively defined operator, and the extremal state $|0, \ldots, 0\rangle$ will become the ground state for our system, associated to the lowest eigenvalue $E_{0, \ldots, 0}=g_{0}^{\prime}$ of $H$. On the other hand, if there is at least one index $j$ for which $\gamma_{j}=-1$, then $H-g_{0}^{\prime}$ will not be positively defined, since the corresponding $j$-th term is of inverted oscillator type, and the state $|0, \ldots, 0\rangle$ will not be a ground state for our system (however it keeps its extremal nature since it is always annihilated by the $n$ operators $\left.B_{j}, j=1, \ldots, n\right)$.

Following [16, 28] it is straightforward to see that, besides the global algebraic structure, there is an intrinsic algebraic structure for our system, characterized by the existing relationship between the Hamiltonian $H$ and the $n$ number operators $N_{k}$ :

$$
H=E\left(N_{1}, \ldots, N_{n}\right)=\sum_{k=1}^{n} \gamma_{k} \omega_{k} N_{k}+g_{0}^{\prime} .
$$

As in the examples discussed in [16, 28], it turns out that this intrinsic algebraic structure is responsible for the specific spectrum of our Hamiltonian. On the other hand, the global algebraic structure arises from the existence of the $n$ independent oscillator modes for $H$, each one characterized by the standard generators $\left\{N_{j}, B_{j}, B_{j}^{\dagger}\right\}, j=1, \ldots, n$. This global behavior allows us to identify in a natural way the extremal state $|0, \ldots, 0\rangle \equiv|\mathbf{0}\rangle$, which plays the role of a ground state although it does not necessarily has a minimum energy eigenvalue. Moreover, the very existence of the extremal state $|\mathbf{0}\rangle$ is guaranteed by a theorem [24] ensuring that if the operators $\left\{B_{1}, \ldots, B_{n}\right\}$ obey the commutation relations given by Eq. (23), then the system of partial differential equations

$$
\left\langle\vec{x}\left|B_{j}\right| 0, \ldots, 0\right\rangle=\left\langle\vec{x}\left|B_{j}\right| \mathbf{0}\right\rangle=0, \quad j=1, \ldots, n,
$$

has the square integrable solution

$$
\phi_{\mathbf{0}}(\vec{x})=\langle\vec{x} \mid \mathbf{0}\rangle=c e^{-\frac{1}{2} a_{i j} x_{i} x_{j}}=c e^{-\frac{1}{2}\left(\vec{x}^{T} \mathbf{a} \vec{x}\right)},
$$


with $c$ being a normalization factor. In this wave function, $\mathbf{a}=\left(a_{i j}\right)$ represents a symmetric matrix whose complex entries are found by solving the system of equations (30), leading to

$$
\mathbf{a} \vec{\alpha}_{j}=\vec{\beta}_{j}, \quad j=1, \ldots, n,
$$

where $\vec{\alpha}_{j}$ and $\vec{\beta}_{j}$ are obtained by expressing $B_{j}$ and $B_{j}^{\dagger}$ as

$$
B_{j}=i \vec{P} \cdot \vec{\alpha}_{j}+\vec{X} \cdot \vec{\beta}_{j}, \quad B_{j}^{\dagger}=-i \vec{\alpha}_{j}^{\dagger} \cdot \vec{P}+\vec{\beta}_{j}^{\dagger} \cdot \vec{X}, \quad j=1, \ldots, n .
$$

The wave functions for the other Fock states can be found from Eq.(26).

\section{Coherent States}

Once our Hamiltonian has been expressed appropriately in terms of annihilation and creation operators, we can develop a similar treatment as for the harmonic oscillator to build up the corresponding coherent states. Here we are going to construct them either as simultaneous eigenstates of the annihilation operators of the system or as the ones resulting from acting the global displacement operator onto the extremal state.

\subsection{Annihilation Operator Coherent States (AOCS)}

In the first place let us look for the annihilation operator coherent states (AOCS) as common eigenstates of the $B_{j}$ 's:

$$
B_{j}\left|z_{1}, \ldots, z_{n}\right\rangle=z_{j}\left|z_{1}, \ldots, z_{n}\right\rangle, \quad z_{j} \in \mathbb{C}, \quad j=1, \ldots, n .
$$

Following a standard procedure, let us expand them in the basis $\left\{\left|n_{1}, \ldots, n_{n}\right\rangle\right\}$ :

$$
\left|z_{1}, \ldots, z_{n}\right\rangle=\sum_{n_{1}, \ldots, n_{n}=0}^{\infty} c_{n_{1}, \ldots, n_{n}}\left|n_{1}, \ldots, n_{n}\right\rangle .
$$

By imposing now that Eq.(34) is satisfied, the following recurrence relationships are obtained,

$$
c_{n_{1}, \ldots, n_{j}, \ldots, n_{n}}=\frac{z_{j}}{\sqrt{n_{j}}} c_{n_{1}, \ldots, n_{j}-1, \ldots, n_{n}}, \quad j=1, \ldots, n,
$$

which, when iterated, lead to

$$
c_{n_{1}, \ldots, n_{j}, \ldots, n_{n}}=\frac{z_{j}^{n_{j}}}{\sqrt{n_{j} !}} c_{n_{1}, \ldots, 0, \ldots, n_{n}}, \quad j=1, \ldots, n .
$$

Hence, it turns out that

$$
c_{n_{1}, \ldots, n_{n}}=\frac{z_{1}^{n_{1}} \ldots z_{n}^{n_{n}}}{\sqrt{n_{1} ! \cdots n_{n} !}} c_{0, \ldots, 0}
$$

where $c_{0, \ldots, 0}$ is to be found from the normalization condition. Thus the normalized AOCS become finally:

$$
\left|z_{1}, \ldots, z_{n}\right\rangle=\exp \left(-\frac{1}{2} \sum_{j=1}^{n}\left|z_{j}\right|^{2}\right) \sum_{n_{1}, \ldots, n_{n}=0}^{\infty} \frac{z_{1}^{n_{1}} \ldots z_{n}^{n_{n}}\left|n_{1}, \ldots, n_{n}\right\rangle}{\sqrt{n_{1} ! \cdots n_{n} !}}
$$

up to a global phase factor. 


\subsection{Displacement Operator Coherent States (DOCS)}

The displacement operator for the $j$-th oscillator mode of the Hamiltonian reads

$$
D_{j}\left(z_{j}\right)=\exp \left(z_{j} B_{j}^{\dagger}-z_{j}^{*} B_{j}\right)
$$

By using the $\mathrm{BCH}$ formula it turns out that

$$
D_{j}\left(z_{j}\right)=\exp \left(-\frac{\left|z_{j}\right|^{2}}{2}\right) \exp \left(z_{j} B_{j}^{\dagger}\right) \exp \left(-z_{j}^{*} B_{j}\right) .
$$

Now, the global displacement operator is given by:

$$
D(\mathbf{z}) \equiv D\left(z_{1}, \ldots, z_{n}\right)=D_{1}\left(z_{1}\right) \cdots D_{n}\left(z_{n}\right)
$$

where $\mathbf{z}$ denotes the complex variables $z_{1}, \ldots, z_{n}$ associated to the $n$ oscillator modes.

Let us obtain now the displacement operator coherent states (DOCS) $|\mathbf{z}\rangle$ from applying $D(\mathbf{z})$ onto the extremal state $|0, \ldots, 0\rangle \equiv|\mathbf{0}\rangle$ :

$$
|\mathbf{z}\rangle=D(\mathbf{z})|\mathbf{0}\rangle=\exp \left(-\frac{1}{2} \sum_{j=1}^{n}\left|z_{j}\right|^{2}\right) \sum_{n_{1}, \ldots, n_{n}=0}^{\infty} \frac{z_{1}^{n_{1}} \ldots z_{n}^{n_{n}}\left|n_{1}, \ldots, n_{n}\right\rangle}{\sqrt{n_{1} ! \cdots n_{n} !}} .
$$

Notice that the AOCS and the DOCS are the same (compare Eqs.(39) and (43)).

\subsection{Coherent state wave functions}

In order to find the wave functions of the coherent states previously derived, we employ that $\left[z_{j} B_{j}^{\dagger}-z_{j}^{*} B_{j}, z_{k} B_{k}^{\dagger}-z_{k}^{*} B_{k}\right]=0 \forall j, k$. Thus:

$$
\begin{aligned}
D(\mathbf{z}) & =\exp \left(z_{1} B_{1}^{\dagger}-z_{1}^{*} B_{1}\right) \cdots \exp \left(z_{n} B_{n}^{\dagger}-z_{n}^{*} B_{n}\right) \\
& =\exp \left[\left(z_{1} B_{1}^{\dagger}+\cdots+z_{n} B_{n}^{\dagger}\right)-\left(z_{1}^{*} B_{1}+\cdots+z_{n}^{*} B_{n}\right)\right] .
\end{aligned}
$$

Using now Eq.(33) we can write

$$
D(\mathbf{z})=e^{-i(\vec{\Gamma} \cdot \vec{P}-\vec{\Sigma} \cdot \vec{X})}=e^{-\frac{i}{2} \vec{\Gamma} \cdot \vec{\Sigma}} e^{i \vec{\Sigma} \cdot \vec{X}} e^{-i \vec{\Gamma} \cdot \vec{P}}=e^{\frac{i}{2} \vec{\Gamma} \cdot \vec{\Sigma}} e^{-i \vec{\Gamma} \cdot \vec{P}} e^{i \vec{\Sigma} \cdot \vec{X}}
$$

where we have employed once again the $\mathrm{BCH}$ formula and we have taken

$$
\vec{\Gamma}=2 \operatorname{Re}\left[z_{1}^{*} \vec{\alpha}_{1}+\ldots+z_{n}^{*} \vec{\alpha}_{n}\right], \quad \vec{\Sigma}=-2 \operatorname{Im}\left[z_{1}^{*} \vec{\beta}_{1}+\ldots+z_{n}^{*} \vec{\beta}_{n}\right] .
$$

Now, it is straightforward to find the wave function for the coherent state $|\mathbf{z}\rangle$,

$$
\phi_{\mathbf{z}}(\vec{x})=\langle\vec{x} \mid \mathbf{z}\rangle=\langle\vec{x}|D(\mathbf{z})| \mathbf{0}\rangle=e^{-\frac{i}{2} \vec{\Gamma} \cdot \vec{\Sigma}} e^{i \vec{\Sigma} \cdot \vec{x}}\left\langle\vec{x}\left|e^{-i \vec{P} \cdot \vec{\Gamma}}\right| \mathbf{0}\right\rangle
$$

Since the operator $\vec{P}$ is the coordinate displacement generator [1], it turns out that

$$
\langle\vec{x}| e^{-i \vec{P} \cdot \vec{\Gamma}}=\langle\vec{x}-\vec{\Gamma}|
$$


so that

$$
\phi_{\mathbf{z}}(\vec{x})=e^{-\frac{i}{2} \vec{\Gamma} \cdot \vec{\Sigma}} e^{i \vec{\Sigma} \cdot \vec{x}}\langle\vec{x}-\vec{\Gamma} \mid \mathbf{0}\rangle=e^{-\frac{i}{2} \vec{\Gamma} \cdot \vec{\Sigma}} e^{i \vec{\Sigma} \cdot \vec{x}} \phi_{\mathbf{0}}(\vec{x}-\vec{\Gamma}) .
$$

A further calculation, using Eq.(31), leads finally to

$$
\phi_{\mathbf{z}}(\vec{x})=e^{-\frac{1}{2}\left(\vec{\Gamma}^{T} \mathbf{a}+i \vec{\Sigma}\right) \cdot \vec{\Gamma}} e^{\left(\vec{\Gamma}^{T} \mathbf{a}+i \vec{\Sigma}\right) \cdot \vec{x}} \phi_{\mathbf{0}}(\vec{x}) .
$$

Once again, it becomes evident that the extremal state is important in our treatment, since its wave function determines the corresponding wave function for any other CS. Moreover, as it can be seen from Eq.(49), the position probability density for the CS $|\mathbf{z}\rangle$ becomes just a displaced version of the corresponding one for the extremal state $|\mathbf{0}\rangle$.

\section{Mathematical and physical properties}

Let us derive next the completeness relationship for the previously derived coherent states. Notice that, from the point of view of the analysis of states in the Hilbert space of the system, this is the most important property which our CS would have [9, 11, 29, 30]. This is the reason why several authors use it as the fourth coherent state definition, considering it as the fundamental one which will survive in time (see e.g. [11]). We are going to calculate as well some important physical quantities in these states.

\subsection{Completeness relationship}

A straightforward calculation leads to:

$$
\begin{aligned}
\left(\frac{1}{\pi}\right)^{n} & \int \ldots \int|\mathbf{z}\rangle\langle\mathbf{z}| d^{2} z_{1} \ldots d^{2} z_{n} \\
\quad= & \sum_{m_{1}, n_{1}, \ldots, m_{n}, n_{n}=0}^{\infty} \frac{\left|m_{1}, \ldots, m_{n}\right\rangle\left\langle n_{1}, \ldots, n_{n}\right|}{\sqrt{m_{1} ! n_{1} ! \cdots m_{n} ! n_{n} !}} \prod_{j=1}^{n}\left(\frac{1}{\pi} \int z_{j}^{m_{j}} z_{j}^{* n_{j}} e^{-\left|z_{j}\right|^{2}} d^{2} z_{j}\right)=\mathbf{1},
\end{aligned}
$$

with 1 being the identity operator. Thus, the coherent states $\{|\mathbf{z}\rangle\}$ form a complete set in the state space of the system (indeed they constitute an overcomplete set [31,32]). This implies that any state can be expressed in terms of our coherent states, in particular, an arbitrary coherent state,

$$
\left|\mathbf{z}^{\prime}\right\rangle=\left(\frac{1}{\pi}\right)^{n} \int \ldots \int|\mathbf{z}\rangle\left\langle\mathbf{z} \mid \mathbf{z}^{\prime}\right\rangle d^{2} z_{1} \ldots d^{2} z_{n},
$$

where the reproducing kernel $\left\langle\mathbf{z} \mid \mathbf{z}^{\prime}\right\rangle$ is given by

$$
\left\langle\mathbf{z} \mid \mathbf{z}^{\prime}\right\rangle=\exp \left[-\frac{1}{2} \sum_{j=1}^{n}\left(\left|z_{j}\right|^{2}-2 z_{j}^{*} z_{j}^{\prime}+\left|z_{j}^{\prime}\right|^{2}\right)\right] .
$$

This means that, in general, our coherent states are not orthogonal to each other. Indeed, notice that inside our infinite set of coherent states only the extremal state of the system, $|\mathbf{0}\rangle \equiv \mid \mathbf{z}=$ $\mathbf{0}\rangle=|0, \ldots, 0\rangle$, is also an eigenstate of the Hamiltonian. 


\subsection{Mean values of some physical quantities in a CS}

Now we can calculate easily the mean values $\left\langle X_{j}\right\rangle_{\mathbf{z}} \equiv\left\langle\mathbf{z}\left|X_{j}\right| \mathbf{z}\right\rangle,\left\langle P_{j}\right\rangle_{\mathbf{z}} \equiv\left\langle\mathbf{z}\left|P_{j}\right| \mathbf{z}\right\rangle, j=1, \ldots, n$, in a given coherent state $|\mathbf{z}\rangle=\left|z_{1}, \ldots, z_{n}\right\rangle$, as well as its mean square deviation in terms of the corresponding results for the extremal state $|\mathbf{0}\rangle$. To do that, let us analyze first how the operators $X_{j}, X_{j}^{2}, P_{j}, P_{j}^{2}$ are transformed under $D(\mathbf{z})$. By using Eqs.(45/46) it is straightforward to show that:

$$
D^{\dagger}(\mathbf{z}) X_{j}^{n} D(\mathbf{z})=\left(X_{j}+\Gamma_{j}\right)^{n}, \quad D^{\dagger}(\mathbf{z}) P_{j}^{n} D(\mathbf{z})=\left(P_{j}+\Sigma_{j}\right)^{n}, \quad n=1,2, \ldots
$$

where we have used that, for an operator $A$ which commutes with $[A, B]$, it turns out that

$$
e^{A} B e^{-A}=B+[A, B] \quad \Rightarrow \quad e^{A} B^{n} e^{-A}=(B+[A, B])^{n}, \quad n=1,2, \ldots
$$

Thus, a straightforward calculation leads to

$$
\left\langle X_{j}\right\rangle_{\mathbf{z}} \equiv\left\langle\mathbf{z}\left|X_{j}\right| \mathbf{z}\right\rangle=\left\langle\mathbf{0}\left|D^{\dagger}(\mathbf{z}) X_{j} D(\mathbf{z})\right| \mathbf{0}\right\rangle=\left\langle X_{j}\right\rangle_{\mathbf{0}}+\Gamma_{j} .
$$

On the other hand,

$$
\left\langle X_{j}^{2}\right\rangle_{\mathbf{z}}=\left\langle X_{j}^{2}\right\rangle_{\mathbf{0}}+2 \Gamma_{j}\left\langle X_{j}\right\rangle_{\mathbf{0}}+\Gamma_{j}^{2}
$$

Hence,

$$
\left(\Delta X_{j}\right)_{\mathbf{z}}^{2}=\left\langle X_{j}^{2}\right\rangle_{\mathbf{z}}-\left\langle X_{j}\right\rangle_{\mathbf{z}}^{2}=\left\langle X_{j}^{2}\right\rangle_{\mathbf{0}}-\left\langle X_{j}\right\rangle_{\mathbf{0}}^{2}=\left(\Delta X_{j}\right)_{\mathbf{0}}^{2} .
$$

Working in a similar way for $P_{j}$, it is obtained

$$
\left\langle P_{j}\right\rangle_{\mathbf{z}}=\left\langle P_{j}\right\rangle_{\mathbf{0}}+\Sigma_{j}, \quad\left\langle P_{j}^{2}\right\rangle_{\mathbf{z}}=\left\langle P_{j}^{2}\right\rangle_{\mathbf{0}}+2 \Sigma_{j}\left\langle P_{j}\right\rangle_{\mathbf{0}}+\Sigma_{j}{ }^{2} .
$$

Then we have as well that

$$
\left(\Delta P_{j}\right)_{\mathbf{z}}^{2}=\left(\Delta P_{j}\right)_{\mathbf{0}}^{2}
$$

i.e., the mean square deviations of $X_{j}$ and $P_{j}$ in the CS $|\mathbf{z}\rangle$ are independent of $\mathbf{z}$.

In order to end up this calculation, the mean values $\left\langle X_{j}\right\rangle_{0},\left\langle P_{j}\right\rangle_{0},\left\langle X_{j}^{2}\right\rangle_{0}$, and $\left\langle P_{j}^{2}\right\rangle_{0}$ for $j=1, \ldots, n$ are required. Let us describe now the procedure to find these $4 n$ quantities. The first $2 n,\left\langle X_{j}\right\rangle_{0},\left\langle P_{j}\right\rangle_{0}$, can be easily found by recalling the definitions of $B_{k}, B_{k}^{\dagger}$ (see section 2.1) and using the fact that their mean values in the extremal state $|\mathbf{0}\rangle$ always vanish for $k=1, \ldots, n$ :

$$
\left\langle B_{k}\right\rangle_{\mathbf{0}}=\left\langle B_{k}^{\dagger}\right\rangle_{\mathbf{0}}=0, \quad k=1, \ldots, n .
$$

This is equivalent to the following linear system of $2 n$ homogeneous equations

$$
f_{k}^{-}\langle\eta\rangle_{\mathbf{0}}=f_{k}^{+}\langle\eta\rangle_{\mathbf{0}}=0, \quad k=1, \ldots, n .
$$

Since the left eigenvectors $f_{k}^{ \pm}, k=1, \ldots, n$, are linearly independent, the only solution for the $2 n$ unknowns $\langle\eta\rangle_{0}$ is the trivial one, i.e., $\left\langle X_{j}\right\rangle_{\mathbf{0}}=\left\langle P_{j}\right\rangle_{\mathbf{0}}=0$. It is worth to point out that this result simplifies Eqs.(56/57/59).

On the other hand, the mean values of the quadratic operators $X_{i}^{2}, P_{i}^{2}, i=1, \ldots, n$, in the extremal state $|\mathbf{0}\rangle$ can be obtained from evaluating the corresponding quantities for the several non-equivalent products of pairs of annihilation $B_{j}$ and creation $B_{k}^{\dagger}$ operators. It is important to mention that these products should have the appropriate order to use the fact that $B_{j}$ annihilates $|\mathbf{0}\rangle$ and $B_{k}^{\dagger}$ annihilates $\langle\mathbf{0}|$ (if the product involves one $B_{j}$ it should be placed to the right while 
if it involves one $B_{k}^{\dagger}$ it should be placed to the left). In general, we will get $n(2 n+1)$ nonequivalent products of pairs of operators $B_{i}, B_{j}^{\dagger}, i, j=1, \ldots, n: n(n+1) / 2$ products of kind $B_{i} B_{j}, j=1, \ldots, n, i \leq j ; n(n+1) / 2$ products of kind $B_{i}^{\dagger} B_{j}^{\dagger}, j=1, \ldots, n, i \leq j ; n^{2}$ products of kind $B_{i}^{\dagger} B_{j}, i, j=1, \ldots, n$. The mean values in the extremal state $|0\rangle$ will lead to an inhomogeneous systems of $n(2 n+1)$ equations, with the same number of unknowns. When solving this system we will get $\left\langle X_{j}^{2}\right\rangle_{0},\left\langle P_{j}^{2}\right\rangle_{0}, j=1, \ldots, n$, and the mean value of any other product of two canonical operators $X_{i}, P_{j}$.

It is customary nowadays to group the mean values of the quadratic products of the operators $X_{i}, P_{j}$ in the coherent state $|\mathbf{z}\rangle$ in a $2 n \times 2 n$ real symmetric matrix $\sigma(\mathbf{z})$, called covariance matrix, whose elements are given by (remember that $\eta=\left(X_{1}, \ldots, X_{n}, P_{1}, \ldots, P_{n}\right)^{T}$ ):

$$
\sigma_{i j}(\mathbf{z})=\frac{1}{2}\left\langle\eta_{i} \eta_{j}+\eta_{j} \eta_{i}\right\rangle_{\mathbf{z}}-\left\langle\eta_{i}\right\rangle_{\mathbf{z}}\left\langle\eta_{j}\right\rangle_{\mathbf{z}}, \quad i, j=1, \ldots 2 n .
$$

A straightforward calculation leads to

$$
\sigma_{i j}(\mathbf{z})=\sigma_{i j}(\mathbf{0})=\frac{1}{2}\left\langle\eta_{i} \eta_{j}+\eta_{j} \eta_{i}\right\rangle_{\mathbf{0}} \equiv \sigma_{i j},
$$

where we have used that $\left\langle\eta_{i}\right\rangle_{\mathbf{0}}=0, i=1, \ldots, 2 n$. The conclusion is that the covariance matrix in our coherent state $|\mathbf{z}\rangle$ is once again independent from $\mathbf{z}$ and depends just of the extremal state $|\mathbf{0}\rangle$. Notice that the number of independent matrix elements $\sigma_{i j}(n(2 n+1))$ coincides with the number of unknowns which are determined from the set of $n(2 n+1)$ independent equations associated to the mean values of the quadratic products of $B_{j}^{\dagger}, B_{k}$ in the extremal state $|\mathbf{0}\rangle$.

Once the covariance matrix is determined, the generalized uncertainty relation can be evaluated [33- 35]

$$
\sigma_{i i} \sigma_{n+i n+i}-\sigma_{i n+i} \geq \frac{1}{4}, \quad i=1, \ldots, n,
$$

which coincides with the Robertson-Schrödinger uncertainty relation (see e.g. [33]).

Let us end up this section by calculating the mean value of the Hamiltonian in a given coherent state $|\mathbf{z}\rangle$. Equation (28) leads to

$$
\langle H\rangle_{\mathbf{z}}=\langle\mathbf{z}|H| \mathbf{z}\rangle=\sum_{k=1}^{n} \gamma_{k} \omega_{k}\left|z_{k}\right|^{2}+g_{0}^{\prime}
$$

In order to get $\left\langle H^{2}\right\rangle_{\mathbf{z}}$, let us notice that

$$
H^{2}=\sum_{j, k=1}^{n} \gamma_{j} \gamma_{k} \omega_{j} \omega_{k} B_{j}^{\dagger} B_{k}^{\dagger} B_{j} B_{k}+\sum_{k=1}^{n} \omega_{k}^{2} B_{k}^{\dagger} B_{k}+2 g_{0}^{\prime} \sum_{k=1}^{n} \gamma_{k} \omega_{k} B_{k}^{\dagger} B_{k}+g_{0}^{\prime 2} .
$$

Thus we get

$$
\left\langle H^{2}\right\rangle_{\mathbf{z}}=\sum_{j, k=1}^{n} \gamma_{j} \gamma_{k} \omega_{j} \omega_{k}\left|z_{j}\right|^{2}\left|z_{k}\right|^{2}+\sum_{k=1}^{n} \omega_{k}^{2}\left|z_{k}\right|^{2}+2 g_{0}^{\prime} \sum_{k=1}^{n} \gamma_{k} \omega_{k}\left|z_{k}\right|^{2}+g_{0}^{\prime 2} .
$$

Hence,

$$
(\Delta H)_{\mathbf{z}}^{2}=\sum_{k=1}^{n} \omega_{k}^{2}\left|z_{k}\right|^{2}
$$

Notice that, for one-dimensional systems $(n=1)$, this expression reduces to the standard one for the harmonic oscillator (see e.g. [1]). 


\subsection{Time evolution of the CS}

Suppose that at $t=0$ our system is in a coherent state $|\mathbf{z}\rangle$. Thus, at a later time $t>0$ the evolved state is found by acting on $|\mathbf{z}\rangle$ with the evolution operator of the system $U(t)=\exp (-i H t)$. By making use of Eq.(29) it turns out that

$$
U(t)=e^{-i g_{0}^{\prime} t} \prod_{k=1}^{n} e^{-i \gamma_{k} \omega_{k} N_{k}}
$$

Hence,

$$
U(t)|\mathbf{z}\rangle=e^{-i g_{0}^{\prime} t}\left|z_{1}(t), \ldots, z_{n}(t)\right\rangle=e^{-i g_{0}^{\prime} t}|\mathbf{z}(t)\rangle,
$$

where $z_{j}(t)=e^{-i \gamma_{j} \omega_{j} t} z_{j}=\left|z_{j}\right| e^{i\left(\theta_{j}-\gamma_{j} \omega_{j} t\right)}$. Equation (70) implies that a coherent state $|\mathbf{z}\rangle$ evolves in time into a new coherent state $|\mathbf{z}(t)\rangle=\left|z_{1}(t), \ldots, z_{n}(t)\right\rangle$, where the $j$-th degree of freedom $z_{j}(t)$ just rotates at its characteristic frequency $\omega_{j}$ (clockwise if $\gamma_{j}=1$ and counterclockwise if $\gamma_{j}=-1$ ).

\subsection{Gazeau-Klauder coherent states}

At this point, it would be interesting to check if our CS belong to the class introduced recently by Gazeau and Klauder [36]. Using their notation, for a system with a Hamiltonian $\mathcal{H}$ such that the ground state energy is zero, the Gazeau-Klauder CS $\{|J, \theta\rangle, J \geq 0,-\infty<\theta<\infty\}$ obey the following properties:

(a) Continuity: $\left(J^{\prime}, \theta^{\prime}\right) \rightarrow(J, \theta) \Rightarrow\left|J^{\prime}, \theta^{\prime}\right\rangle \rightarrow|J, \theta\rangle$.

(b) Resolution of unity: $\mathbf{1}=\int|J, \theta\rangle\langle J, \theta| d \mu(J, \theta)$.

(c) Temporal stability: $e^{-i \mathcal{H} t}|J, \theta\rangle=|J, \theta+\omega t\rangle, \omega=$ constant.

(d) Action identity: $\langle J, \theta|\mathcal{H}| J, \theta\rangle=\omega J$.

Concerning the first property, it is straightforward to check that our coherent states given in Eq.(43) are such that $\left|\mathbf{z}^{\prime}\right\rangle \rightarrow|\mathbf{z}\rangle$ as $\mathbf{z}^{\prime} \rightarrow \mathbf{z}$, i.e., they are continuous in $\mathbf{z}$. As for the second and third properties, both were explicitly proven in sections 4.1 and 4.3 respectively. It remains just to analyze if it is valid the action identity given in (d). Let us notice first of all that it is valid for each partial Hamiltonian $H_{k}=\gamma_{k} \omega_{k} N_{k}$ of our system,

$$
\left\langle\mathbf{z}\left|H_{k}\right| \mathbf{z}\right\rangle=\gamma_{k} \omega_{k}\left|z_{k}\right|^{2}
$$

which is time-independent. Therefore, property (d) becomes valid for each degree of freedom separately and thus it is valid for our global system with the natural identification $J_{k}=\left|z_{k}\right|^{2}$, $\theta_{k}=\arg \left(z_{k}\right)$ so that

$$
\left\langle\mathbf{z}\left|\left(H-g_{0}^{\prime}\right)\right| \mathbf{z}\right\rangle=\sum_{k=1}^{n} \gamma_{k} \omega_{k} J_{k} .
$$

We conclude that our CS of Eq.(43) become as well an $n$-dimensional generalization of the Gazeau-Klauder CS if we express each complex component $z_{k}$ of $\mathbf{z}$ in its polar form (the polar coordinates essentially coincide with the canonical action-angle variables for the corresponding classical system). 


\section{Asymmetric Penning trap coherent states}

Let us apply now the previous technique to the asymmetric Penning trap. Such an arrangement can be used to control some quantum mechanical phenomena [37] as well as to perform highprecision measurements of fundamental properties of particles. Moreover, it is a quite natural system to analyze the decoherence taking place due to the unavoidable interaction of the system with its environment [38, 39]. Since the asymmetric Penning trap becomes the ideal one when the asymmetry parameter vanishes [40-42], it will be straightforward to compare these results with those recently obtained for the ideal Penning trap [16] (see also [35, 43, 44]).

The Hamiltonian of a charged particle with mass $m$ and charge $q$ in an asymmetric Penning trap reads

$$
H=\frac{\vec{P}^{2}}{2 m}+\frac{\omega_{c}}{2}\left(X P_{y}-Y P_{x}\right)+\frac{m}{2}\left(\omega_{x}^{2} X^{2}+\omega_{y}^{2} Y^{2}+\omega_{z}^{2} Z^{2}\right),
$$

$\omega_{c}=q B / m$ and $\omega_{z}$ being the cyclotron and axial frequencies respectively, and the frequencies $\omega_{x}, \omega_{y}$ are given by

$$
\omega_{x}^{2}=\frac{\omega_{c}^{2}}{4}-\frac{\omega_{z}^{2}}{2}(1+\varepsilon), \quad \omega_{y}^{2}=\frac{\omega_{c}^{2}}{4}-\frac{\omega_{z}^{2}}{2}(1-\varepsilon)
$$

where $|\varepsilon|<1$ is the real asymmetry parameter and we are denoting $\vec{P}=\left(P_{x}, P_{y}, P_{z}\right)^{T}, \vec{X}=$ $(X, Y, Z)^{T}$. Without loosing generality [16], from now on we will assume that $m=1$.

As it was seen at section 2, the main role in our treatment is played by the matrix $\Lambda$ such that $[i H, \eta]=\Lambda \eta$. We choose here $\eta=\left(X, Y, P_{x}, P_{y}, Z, P_{z}\right)^{T}$ so that

$$
\boldsymbol{\Lambda}=\left(\begin{array}{cccccc}
0 & -\omega_{c} / 2 & 1 & 0 & 0 & 0 \\
\omega_{c} / 2 & 0 & 0 & 1 & 0 & 0 \\
-\omega_{x}^{2} & 0 & 0 & -\omega_{c} / 2 & 0 & 0 \\
0 & -\omega_{y}^{2} & \omega_{c} / 2 & 0 & 0 & 0 \\
0 & 0 & 0 & 0 & 0 & 1 \\
0 & 0 & 0 & 0 & -\omega_{z}^{2} & 0
\end{array}\right)
$$

The eigenvalues $(\lambda)$ of $\Lambda$ are

$$
\begin{aligned}
& \lambda_{1}=\frac{i \omega_{c}}{2} \sqrt{2-\delta+R}=i \omega_{1}, \quad \lambda_{2}=\frac{i \omega_{c}}{2} \sqrt{2-\delta-R}=i \omega_{2}, \\
& \lambda_{3}=i \omega_{z}=i \omega_{3}, \quad R=\sqrt{4(1-\delta)+\delta^{2} \varepsilon^{2}}, \quad 0<\delta=\frac{2 \omega_{z}^{2}}{\omega_{c}^{2}}<1,
\end{aligned}
$$

and their corresponding complex conjugate. The right $(u)$ and left $(f)$ eigenvectors of $\Lambda$ become

$$
\begin{aligned}
& u_{1}^{+}=s_{1}\left(\frac{4}{\omega_{c}(\delta \varepsilon+R)},-\frac{i}{\omega_{1}} \frac{2+\delta \varepsilon+R}{\delta \varepsilon+R}, \frac{i \omega_{c}}{2 \omega_{1}} \frac{2(1-\delta)-\delta \varepsilon+R}{\delta \varepsilon+R}, 1,0,0\right)^{T}, \\
& u_{2}^{+}=s_{2}\left(\frac{4}{\omega_{c}(\delta \varepsilon-R)},-\frac{i}{\omega_{2}} \frac{2+\delta \varepsilon-R}{\delta \varepsilon-R}, \frac{i \omega_{c}}{2 \omega_{2}} \frac{2(1-\delta)-\delta \varepsilon-R}{\delta \varepsilon-R}, 1,0,0\right)^{T}, \\
& u_{3}^{+}=s_{3}\left(0,0,0,0,-\frac{i}{\omega_{z}}, 1\right)^{T},
\end{aligned}
$$




$$
\begin{aligned}
& f_{1}^{+}=t_{1}\left(\frac{\omega_{c}}{4}(R-\delta \varepsilon), \frac{i \omega_{c}^{2}}{8 \omega_{1}}[2(1-\delta)+\delta \varepsilon+R],-\frac{i \omega_{c}}{4 \omega_{1}}(2-\delta \varepsilon+R), 1,0,0\right) \\
& f_{2}^{+}=t_{2}\left(\frac{\omega_{c}}{4}(-R-\delta \varepsilon), \frac{i \omega_{c}^{2}}{8 \omega_{2}}[2(1-\delta)+\delta \varepsilon-R],-\frac{i \omega_{c}}{4 \omega_{2}}(2-\delta \varepsilon-R), 1,0,0\right), \\
& f_{3}^{+}=t_{3}\left(0,0,0,0, i \omega_{z}, 1\right)
\end{aligned}
$$

where $s_{j}, t_{j} \in \mathbb{C}, j=1,2,3$. The requirement that the right and left eigenvectors be dual to each other implies

$$
s_{1}=\frac{1}{4 t_{1}}\left(1+\frac{\delta \varepsilon}{R}\right), \quad s_{2}=\frac{1}{4 t_{2}}\left(1-\frac{\delta \varepsilon}{R}\right), \quad s_{3}=\frac{1}{2 t_{3}} .
$$

On the other hand, up to some phase factors, the condition imposed by Eqs.(10]11) leads to,

$$
t_{1}=\frac{1}{\sqrt{2 i\left(f_{1 a} f_{1 c}-f_{1 b}\right)}}, \quad t_{2}=\frac{1}{\sqrt{2 i\left(f_{2 b}-f_{2 a} f_{2 c}\right)}}, \quad t_{3}=\frac{1}{\sqrt{2 \omega_{3}}},
$$

where we are denoting $f_{1}^{+}=t_{1}\left(f_{1 a}, f_{1 b}, f_{1 c}, 1,0,0\right), f_{2}^{+}=t_{2}\left(f_{2 a}, f_{2 b}, f_{2 c}, 1,0,0\right)$ in order to simplify the notation (compare Eq.(75)). Moreover, the crucial signs for us to conclude that our asymmetric Penning trap Hamiltonian is not positively defined become:

$$
\gamma_{1}=1, \quad \gamma_{2}=-1, \quad \gamma_{3}=1
$$

Thus, our annihilation operators take the form (see Eq.(22)):

$$
\begin{aligned}
& B_{1}=L_{1}^{-}=t_{1}\left(f_{1 a} X-f_{1 b} Y-f_{1 c} P_{x}+P_{y}\right), \\
& B_{2}=L_{2}^{+}=t_{2}\left(f_{2 a} X+f_{2 b} Y+f_{2 c} P_{x}+P_{y}\right), \\
& B_{3}=L_{3}^{-}=t_{3}\left(-i \omega_{3} Z+P_{z}\right) .
\end{aligned}
$$

From these operators and their hermitian conjugates, it is straightforward to identify the $\alpha_{j}$ and $\beta_{j}, j=1,2,3$ which allow us to find the matrix a such that $\mathbf{a} \alpha_{j}=\beta_{j}$. Its matrix elements $a_{i j}$ become now:

$$
\begin{aligned}
& a_{11}=-i \frac{f_{1 a}-f_{2 a}}{f_{1 c}+f_{2 c}}, \quad a_{12}=a_{21}=i \frac{f_{1 b}+f_{2 b}}{f_{1 c}+f_{2 c}}, \quad a_{22}=i \frac{f_{1 c} f_{2 b}-f_{2 c} f_{1 b}}{f_{1 c}+f_{2 c}} \\
& a_{33}=\omega_{3}, \quad a_{13}=a_{31}=a_{23}=a_{32}=0 .
\end{aligned}
$$

It can be shown that $a_{11}, a_{22}, a_{33} \in \mathbb{R}^{+}$while $a_{12}$ is purely imaginary. Thus the extremal state wave function of Eq. (31) acquires the form:

$$
\phi_{\mathbf{0}}(\vec{x})=c \exp \left(-\frac{1}{2} a_{11} x^{2}-\frac{1}{2} a_{22} y^{2}-a_{12} x y\right) \exp \left(-\frac{1}{2} a_{33} z^{2}\right) .
$$

The associated eigenvalue becomes $E_{0,0,0}=\left(\omega_{1}-\omega_{2}+\omega_{3}\right) / 2$.

Concerning the coherent states $\left|z_{1}, z_{2}, z_{3}\right\rangle$, the general treatment developed in section 3 is straightforwardly applicable, and their explicit expressions are given by Eq.(43) with $n=3$. Their corresponding wave functions are given by

$$
\phi_{z}(\vec{x})=\langle\vec{x} \mid \mathbf{z}\rangle=e^{-i \vec{\Gamma} \cdot \vec{\Sigma} / 2} e^{i \vec{\Sigma} \cdot \vec{x}} \phi_{0}\left(x-\Gamma_{1}, y-\Gamma_{2}, z-\Gamma_{3}\right),
$$


where

$$
\vec{\Gamma}=2\left(\begin{array}{c}
i t_{1} f_{1 c} \operatorname{Re}\left[z_{1}\right]-i t_{2} f_{2 c} \operatorname{Re}\left[z_{2}\right] \\
-t_{1} \operatorname{Im}\left[z_{1}\right]-t_{2} \operatorname{Im}\left[z_{2}\right] \\
-t_{3} \operatorname{Im}\left[z_{3}\right]
\end{array}\right), \vec{\Sigma}=2\left(\begin{array}{c}
t_{1} f_{1 a} \operatorname{Im}\left[z_{1}\right]+t_{2} f_{2 a} \operatorname{Im}\left[z_{2}\right] \\
-i t_{1} f_{1 b} \operatorname{Re}\left[z_{1}\right]+i t_{2} f_{2 b} \operatorname{Re}\left[z_{2}\right] \\
t_{3} \omega_{3} \operatorname{Re}\left[z_{3}\right]
\end{array}\right) .
$$

The mean values $\left\langle X_{j}\right\rangle_{\mathbf{z}},\left\langle P_{j}\right\rangle_{\mathbf{z}}$, immediately follow from Eqs. (56, 59) with $\left\langle X_{j}\right\rangle_{\mathbf{0}}=\left\langle P_{j}\right\rangle_{\mathbf{0}}=$ 0, i.e.,

$$
\left\langle X_{j}\right\rangle_{\mathbf{z}}=\Gamma_{j}, \quad\left\langle P_{j}\right\rangle_{\mathbf{z}}=\Sigma_{j}, \quad j=1,2,3 .
$$

As for the mean values of the quadratic operators in the extremal state, we have solved the system of equations arising from the null mean values of the products of pairs of annihilation $B_{j}$ and creation $B_{k}^{\dagger}$ operators. We get

$$
\begin{aligned}
\left\langle X^{2}\right\rangle_{\mathbf{0}} & =\frac{1}{2 a_{11}}, & \left\langle P_{x}^{2}\right\rangle_{\mathbf{0}} & =\frac{1}{2}\left(a_{11}-\frac{a_{12}^{2}}{a_{22}}\right), \\
\left\langle Y^{2}\right\rangle_{\mathbf{0}} & =\frac{1}{2 a_{22}}, & \left\langle P_{y}^{2}\right\rangle_{\mathbf{0}} & =\frac{1}{2}\left(a_{22}-\frac{a_{12}^{2}}{a_{11}}\right), \\
\left\langle Z^{2}\right\rangle_{\mathbf{0}} & =\frac{1}{2 a_{33}}, & \left\langle P_{z}^{2}\right\rangle_{\mathbf{0}} & =\frac{1}{2} a_{33},
\end{aligned}
$$

and the crossed products

$$
\begin{aligned}
& \left\langle X P_{x}\right\rangle_{\mathbf{0}}=\frac{i}{2}, \quad\left\langle X P_{y}\right\rangle_{\mathbf{0}}=\frac{i}{2} \frac{a_{12}}{a_{11}}, \quad\left\langle X P_{z}\right\rangle_{\mathbf{0}}=0, \\
& \left\langle Y P_{x}\right\rangle_{\mathbf{0}}=\frac{i}{2} \frac{a_{12}}{a_{22}}, \quad\left\langle Y P_{y}\right\rangle_{\mathbf{0}}=\frac{i}{2}, \quad\left\langle Y P_{z}\right\rangle_{\mathbf{0}}=0, \\
& \left\langle Z P_{x}\right\rangle_{\mathbf{0}}=0, \quad\left\langle Z P_{y}\right\rangle_{\mathbf{0}}=0, \quad\left\langle Z P_{z}\right\rangle_{\mathbf{0}}=\frac{i}{2} .
\end{aligned}
$$

Therefore, using Eqs.(58, 60) we get the Heisenberg uncertainty relationships

$$
\begin{aligned}
(\Delta X)_{\mathbf{z}}^{2}\left(\Delta P_{x}\right)_{\mathbf{z}}^{2} & =(\Delta Y)_{\mathbf{z}}^{2}\left(\Delta P_{y}\right)_{\mathbf{z}}^{2}=\frac{1}{4}\left(1+\frac{\left|a_{12}\right|^{2}}{a_{11} a_{22}}\right) \geq \frac{1}{4}, \\
(\Delta Z)_{\mathbf{z}}^{2}\left(\Delta P_{z}\right)_{\mathbf{z}}^{2} & =\frac{1}{4},
\end{aligned}
$$

while Eq. (69) with $n=3$ gives the mean square deviation for the Hamiltonian.

Once we have calculated the mean values of the quadratic products given in Eqs. 85186), it is straightforward to evaluate the covariance matrix elements of Eq.(64). With the ordering $\eta=\left(X, Y, P_{x}, P_{y}, Z, P_{z}\right)^{T}$, it is obtained:

$$
\sigma=\left(\begin{array}{cccccc}
(\Delta X)_{\mathbf{0}}^{2} & 0 & 0 & \frac{i a_{12}}{2 a_{11}} & 0 & 0 \\
0 & (\Delta Y)_{\mathbf{0}}^{2} & \frac{i a_{12}}{2 a_{22}} & 0 & 0 & 0 \\
0 & \frac{i a_{12}}{2 a_{22}} & \left(\Delta P_{x}\right)_{\mathbf{0}}^{2} & 0 & 0 & 0 \\
\frac{i a_{12}}{2 a_{11}} & 0 & 0 & \left(\Delta P_{y}\right)_{\mathbf{0}}^{2} & 0 & 0 \\
0 & 0 & 0 & 0 & (\Delta Z)_{\mathbf{0}}^{2} & 0 \\
0 & 0 & 0 & 0 & 0 & \left(\Delta P_{z}\right)_{\mathbf{0}}^{2}
\end{array}\right) .
$$


Notice that this covariance matrix is non-diagonal. However, since $\sigma_{13}=\sigma_{24}=\sigma_{56}=0$, it turns out that the generalized uncertainty relations of Eq. (65) reduce to the Heisenberg uncertainty relations given in Eq. (87).

A plot of $(\Delta X)_{\mathbf{z}}\left(\Delta P_{x}\right)_{\mathbf{z}}$ as a function of the parameters $\varepsilon$ and $\delta$ is given in Fig.1. As it can be seen from Eqs. 80 87) and from Fig.1, the coherent states minimize the Heisenberg uncertainty relationship for $\varepsilon=0$, which coincides with the results recently obtained for the ideal Penning trap [16]. However, for $\varepsilon \neq 0$ it turns out that $(\Delta X)_{\mathbf{z}}\left(\Delta P_{x}\right)_{\mathbf{z}}>1 / 2$. Notice that the same plot will appear for the uncertainty product $(\Delta Y)_{\mathbf{z}}\left(\Delta P_{y}\right)_{\mathbf{z}}$.

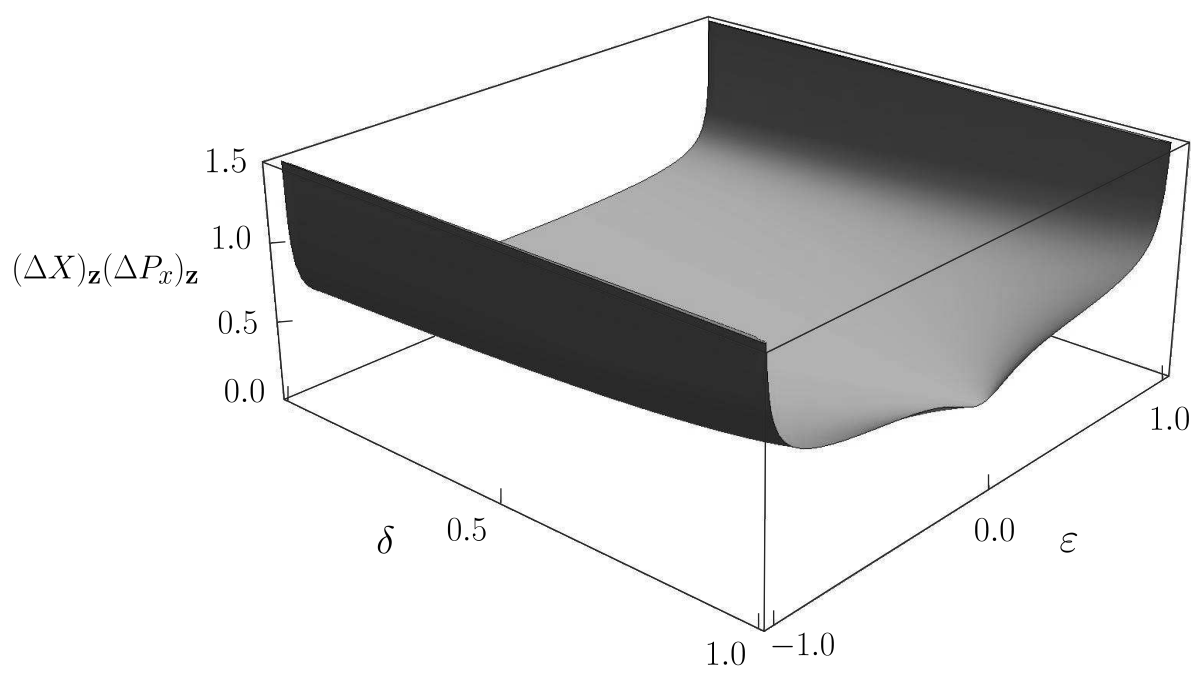

Figure 1: Heisenberg uncertainty relationship $(\Delta X)_{\mathbf{z}}\left(\Delta P_{x}\right)_{\mathbf{z}}$ for the asymmetric Penning trap coherent states as function of the real dimensionless parameters $|\varepsilon|<1,0<\delta<1$.

\section{Concluding remarks}

In this work we have proposed a systematic technique to find the CS for systems governed by quadratic Hamiltonians in the trap regime. To do this, we introduced a prescription to identify in a simple way the appropriate ladder operators which play the same role as the annihilation and creation operators for the 1-dimensional harmonic oscillator. These operators allowed us to generate the eigenvectors and eigenvalues for the Hamiltonian departing from the extremal state, the analogue of the ground state although it is not necessarily an eigenstate associated to the lowest possible eigenvalue. The explicit expression for the extremal state wave function was as well explicitly calculated.

For systems governed by this kind of Hamiltonians the two algebraic CS definitions (either as simultaneous eigenstates of the annihilation operators or as resulting from the action of the displacement operator onto the extremal state) lead to the same set of states. The explicit expression for the corresponding wave functions has been also derived.

We have calculated explicitly the mean values of the position and momentum operators in an arbitrary coherent state. Moreover, we have provided as well a prescription to obtain 
algebraically, by solving a linear systems of equations, the mean values of the quadratic products of these operators in the CS.

Through this method we have found the asymmetric Penning trap coherent states and we have explored some of their physical properties. In particular, it is worth to point out that, in general, they do not minimize the Heisenberg uncertainty relationship. The differences from the minimum are induced by the deviations of the axial symmetry which the ideal Penning trap has (measured by the asymmetry parameter $\varepsilon$ ).

\section{Acknowledgments}

The authors acknowledge the support of Conacyt.

\section{References}

[1] C. Cohen-Tannodji, B. Diu, F. Laloë, Quantum Mechanics, Wiley-Interscience, New York (1977)

[2] R.J. Glauber, Photon correlations, Phys. Rev. Lett. 10 (1963) 84-86

[3] R.J. Glauber, Coherent and incoherent states of the radiation field, Phys. Rev. 131 (1963) 2766-2788

[4] V. Bargmann, On a Hilbert space of analytic functions and an associated integral transform. Part I, Commun. Pure App. Math. 14 (1961) 187-214

[5] A.O. Barut, L. Girardello, New “coherent states"associated with non-compact grups, Commun. Math. Phys. 21 (1971) 41-55

[6] A.M. Perelomov, Coherent states for arbitrary lie group, Commun. Math. Phys. 26 (1972) 222-236

[7] V.V. Dodonov, E.V. Kurmyshev, V.I. Man'ko, Generalized uncertainty relation and correlated coherent states, Phys. Lett. A 79 (1980) 150-152

[8] A. Perelomov, Generalized coherent states and their applications, Springer-Verlag, Heidelberg (1986)

[9] J.R. Klauder, B.S. Skagerstam Eds., Coherent states. Applications in physics and mathematical physics, World Scientific, Singapore (1985)

[10] W.M Zhang, D.H. Feng, R. Gilmore, Coherent states: Theory and some applications, Rev. Mod. Phys. 62 (1990) 867-928

[11] S.T. Ali, J.P. Antoine, J.P. Gazeau, Coherent states, wavelets and their generalizations, Springer-Verlag, New York (2000)

[12] C. Quesne, Generalized coherent states associated with the $C_{\lambda}$ extended oscillator, Ann. Phys. 293 (2001) 147-188 
[13] V.V. Dodonov, Nonclassical states in quantum optics: a squeezed review of the first 75 years, J. Opt. B 4 (2002) R1-R33

[14] D.J. Fernández C., Coherent states for one-dimensional systems, AIP Conf. Proc. 809 (2006) 80-85

[15] H. Friedrich, Theoretical atomic physics, Springer-Verlag, Heidelberg (2006)

[16] D.J. Fernández C., M. Velázquez, Coherent states approach to Penning trap, J. Phys. A: Math. Theor. 42 (2009) 085304

[17] V.V. Dodonov, V.I. Manko, Invariants and the evolution of nonstationary quantum systems, ed. M.A. Markov, Nova Science, New York (1989)

[18] M. Velázquez, Estados coherentes para Hamiltonianos cuadráticos tridimensionales con simetría axial, M.Sc. Thesis, Cinvestav (2007)

[19] B. Mielnik, A. Ramírez, Ion traps: some semiclassical observations, Phys. Scr. 82 (2010) 055002

[20] B. Mielnik, A. Ramírez, Magnetic operations: a little fuzzy physics?, preprint Cinvestav (2010) arXiv:1006.1944v1 [quant-ph]

[21] B. Mielnik, O. Rosas-Ortiz, Factorization: little or great algorithm?, J. Phys. A: Math. Gen. 37 (2004) 10007-10035

[22] D.J. Fernández C., N. Fernández-García, Higher-order supersymmetric quantum mechanics, AIP Conf. Proc. 744 (2005) 236-273

[23] J.V. Moloney, F.H.M. Faisal, Time-dependent theory of non-hermitian Schrödinger equation: application to multiphoton-induced ionization decay of atoms, J. Phys. B 14 (1981) 3603-3620

[24] B. Mielnik, D.J. Fernández C., An electron trapped in a rotating magnetic field, J. Math. Phys. 30 (1989) 537-549

[25] D.J. Fernández C., Semiclassical resonance in rotating magnetic fields, Acta Phys. Polon. B21 (1990) 589-601

[26] S.G. Cruz y Cruz, Esquemas cuánticos de Floquet: espectros y operaciones, Ph.D. Thesis, Cinvestav (2005)

[27] S. Cruz y Cruz, B. Mielnik, The parity phenomenon of the Floquet spectra, Phys. Lett. A 352 (2006) 36-40

[28] D.J. Fernández C., V. Hussin, O. Rosas-Ortiz, Coherent states for Hamiltonians generated by supersymmetry, J. Phys. A: Math. Theor. 40 (2007) 6491-6511

[29] J.R. Klauder, Continuous-representation theory. I. Postulates of continuous representation theory, J. Math. Phys. 4 (1963) 1055-1058 
[30] J.R. Klauder, Continuous-representation theory. II. Generalized relation between quantum and classical dynamics, J. Math. Phys. 4 (1963) 1058-1073

[31] K.E. Cahill, Coherent-state representations for the photon density operator, Phys. Rev. 138 (1965) B1566-B1576

[32] V. Bargmann, P. Butera, L. Girardello, J.R. Klauder, On the completeness of the coherent states, Rep. Math. Phys. 2 (1971) 221-228

[33] S. Hacyan, Squeezed states and uncertainty relations in rotating frames and Penning traps, Phys. Rev. A 53 (1996) 4481-4487

[34] G. Adesso, F. Illuminati, Entanglement in continuous-variable systems: recent advances and current perspectives, J. Phys. A: Math. Theor. 40 (2007) 7821-7880

[35] M. Genkin, E. Lindroth, On the Penning trap coherent states, J. Phys. A: Math. Theor. 42 (2009) 275305

[36] J.P. Gazeau, J.R. Klauder, Coherent states for systems with discrete and continuous spectrum, J. Phys. A: Math. Gen. 32 (1999) 123-132

[37] D.J. Fernández C., B. Mielnik, Controlling quantum motion, J. Math. Phys. 35 (1994) 2083-2104

[38] L.S. Brown, G. Gabrielse, Geonium theory. Physics of a single electron or ion in a Penning trap, Rev. Mod. Phys. 58 (1986) 233-311

[39] M. Genkin, E. Lindroth, Environmental effects on the phase space dynamics and decoherence time scale of a charged particle in a Penning trap, J. Phys. A: Math. Theor. 42 (2009) 385302

[40] D.J. Fernández C., L.M. Nieto, Penning trap from the phase space quantum mechanics point of view, Phys. Lett. A 157 (1991) 315-319

[41] D.J. Fernández C., Transformations of a wave packet in a Penning trap, Nuovo Cim. 107B (1992) 885-893

[42] D.J. Fernández C., N. Bretón, Is there a prescribed parameter's space for the adiabatic geometric phase?, Europhys. Lett. 21 (1993) 147-152

[43] O.V. Manko, Symplectic tomography of nonlinear coherent states of a trapped ion, Phys. Lett. A 228 (1997) 29-35

[44] O. Castaños, S. Hacyan, R. López-Peña, V.I. Manko, Schrödinger cat states in a Penning trap, J. Phys. A: Math. Gen. 31 (1998) 1227-1237 